\title{
Collision Detect and Avoidance Media Access Mechanism for Next Generation 802.11ax Networks
}

\author{
Rung-Shiang Cheng \\ Department of Computer and Communication \\ Kun Shan University \\ Tainan, Taiwan (R.O.C) \\ Email: rscheng@mail.ksu.edu.tw
}

\author{
Chung-Ming Huang \\ Department of Computer Science and Information \\ Engineering \\ National Cheng Kung University \\ Tainan, Taiwan (R.O.C) \\ Email: huangcm@locust.csie.ncku.edu.tw
}

\begin{abstract}
IEEE is making an 802.11ax protocol for nextgeneration wireless network. The goal of 802.11ax is to improve the networking performance of individual device, especially those mobile stations with numerous access networks in high-density wireless network. However, traditional 802.11 standards have the problem of poor transmission efficiency because traditional standards for wireless network enhance the overall wireless network volume and are easy to be implemented. When there are numerous wireless networking devices existing in a congested network to continuously conduct random access procedure, the success rate of channel access might drastically drop, leading to problems such as data error, loss, latency, energy consumption, etc.; to improve this problem, when designing message processing mechanism in physical layers, we should also consider the problem of media access control. Hence, this paper proposes a collision detection and avoidance media access mechanism for next-generation WiFi communications. The proposed algorithm leverages the ability of underlying random access to compatible legacy WiFi and identifies available slot-time to avoid channel collision of the access network. The algorithm's performance is evaluated by the simulation which demonstrates significant improvement in terms of channel efficiency as well as transmission delay. The results confirm that the proposed CSMA/CDA scheme provides a better performance than the legacy CSMA/CA in dense WiFi networks.
\end{abstract}

Keywords-IEEE 802.11; DCF; MAC; CSMA/CA

\section{INTRODUCTION}

Wireless technology is one of the most popular ways to connect the end-device and Internet. Wireless access gives mobility to end-devices. There is no need to organize a wired infrastructure for the end-devices, and also the number of enddevice may vary. Recently, the market share of smartphones, tablet computers and so on with $3 \mathrm{G} / \mathrm{LTE}$ adapter and WiFi adapter has been growing rapidly. WiFi technology has become ubiquitous and continues to be one of the fastest growing areas within the field of wireless communications. Nowadays, WiFi is recognized as one of the most popular communication technology. Most of modern laptops and mobile phones are WiFi-capable.

WiFi is already prevalent in last-mile access networks. Since the commercial adoption of $802.11 \mathrm{~b}$ in 1999 , WiFi speeds have increased from $11 \mathrm{Mbps}$ to a maximum of 600

*Corresponding author: Chung-Ming Huang; E-mail:

huangcm@locust.csie.ncku.edu.tw
Mbps for 802.11n. However, applications including high definition streaming video and instantaneous data transfer demand further levels of performance. The emerging IEEE 802.11 ac standard has advantage of wider available bandwidth and lower interference levels at $5 \mathrm{GHz}$. Up to $6.77 \mathrm{Gbps}$ throughput can be achieved by utilizing a maximum of 160 $\mathrm{MHz}$ channel bandwidth, 8 spatial streams, and 256 quadrature amplitude modulation (QAM). Table I summarizes the evolution of the IEEE 802.11 standards [1].

On the other hand, the advantages of IEEE 802.11 cause some difficulties. In the case of infrastructure mode wireless local area network (WLAN), mobile stations (STAs) that associate to the same access points (APs) have to compete for the shared bandwidth with many traffic sources. A bandwidth of one source can be affected by traffic usage of others. Hence, the bandwidth not only depends on the number of STAs but also the difference between their bit rates. High-speed STA bandwidth can be significantly reduced by low-speed STA because of the increasing latency media access, as low-speed STA requires more time to send data frame. The result is that the total capacity of WLAN will be reduced.

Poor WiFi performance is often attributed to wireless interference in heavy loaded scenarios. While a lot of researches have been conducted to explore wireless interference in a theoretical context, real network deployments are yet to gain from it. In this work, we introduce a technique to reduce the channel interference between mobile nodes. The goal is to execute it in the most unobtrusive fashion when possible: (1) Compatible with legacy WiFi media access control mechanism; (2) Promote network performance and reduce meaningless power-consumption. To achieve these goals, this paper proposes a collision detection and avoidance scheme for distributed random access wireless networks named CSMA/CDA.

The proposed CSMA/CDA scheme is designed for nextgeneration IEEE 802.11ax [2] DCF networks to increase the channel efficiency of the network. To our knowledge, this is the first algorithm designed for a contention-based coordination network. In the proposed scheme, when wireless devices require channel resource, it would first acquire channel resource via Random Access (RA) procedure. When transmitting data sequentially, it would insert a Random 
TABLE II. EVOLUTION OF THE IEEE 802.11 STANDARD [1]

\begin{tabular}{|c|c|c|c|c|c|c|c|}
\hline 802.11 Protocol & Year Released & $\begin{array}{l}\text { Frequency } \\
\text { Band }\end{array}$ & $\begin{array}{l}\text { Channel } \\
\text { Widths }\end{array}$ & $\begin{array}{c}\text { Highest Single Stream } \\
\text { Data Rate }\end{array}$ & Modulation Type & $\begin{array}{c}\text { MIMO } \\
\text { Streams }\end{array}$ & $\begin{array}{c}\text { Highest Aggregate } \\
\text { Data Rate }\end{array}$ \\
\hline 802.11 legacy & 1997 & $2.4 \mathrm{G} \mathrm{Hz}$ & $20 \mathrm{MHZ}$ & $2 \mathrm{Mbps}$ & DBPSK,DQPSK & 1 & $2 \mathrm{Mbps}$ \\
\hline $802.11 \mathrm{a}$ & 1999 & $5 \mathrm{GHz}$ & $20 \mathrm{MHZ}$ & $54 \mathrm{Mbps}$ & $\begin{array}{c}\text { BPSK,QPSK, 16-QAM, } \\
\text { 64-QAM }\end{array}$ & 1 & $54 \mathrm{Mbps}$ \\
\hline $802.11 \mathrm{~b}$ & 1999 & $2.4 \mathrm{GHz}$ & $20 \mathrm{MHZ}$ & $11 \mathrm{Mbps}$ & $\mathrm{CCK}$ & 1 & $11 \mathrm{Mbps}$ \\
\hline $802.11 \mathrm{~g}$ & 2003 & $2.4 \mathrm{GHz}$ & $20 \mathrm{MHZ}$ & $54 \mathrm{Mbps}$ & $\begin{array}{c}\text { BPSK,QPSK,16-QAM, } \\
\text { 64-QAM } \\
\end{array}$ & 1 & $54 \mathrm{Mbps}$ \\
\hline $802.11 \mathrm{n}$ & 2009 & 2.4 and $5 \mathrm{GHz}$ & $20,40 \mathrm{MHZ}$ & $150 \mathrm{Mbps}$ & $\begin{array}{c}\text { BPSK,QPSK,16-QAM, } \\
\text { 64-QAM } \\
\end{array}$ & 4 & $600 \mathrm{Mbps}$ \\
\hline $802.11 \mathrm{ac}$ & 2012 & $5 \mathrm{GHz}$ & $\begin{array}{c}20,40,80,160 \\
\text { MHZ }\end{array}$ & 866.7 Mbps & $\begin{array}{c}\text { BPSK,QPSK,16-QAM, } \\
\text { 64-QAM,256-QAM }\end{array}$ & 8 & $6.77 \mathrm{Gbps}$ \\
\hline
\end{tabular}

Register Number in the Header of the data packet and utilize this number to mark preselected random slot, letting AP announce the preselected time slot of this mobile station for next transmission. This assists mobile station in collision detection and avoidance to increase the performance of the overall and individual mobile station.

The remainder of this paper is organized as follows. Section 2 gives an overview of the legacy media access in $\mathrm{WiFi}$ technology. Section 3 describes the proposed CSMA/CDA scheme. Results from performance are presented and discussed in Section 4. Finally, Section 5 presents the conclusions.

\section{LEGACY IEEEE 802.11 WIRELESS COMMUNICATIONS}

The IEEE 802.11 standard defines two types of services, namely a contention-free polling-based point coordination function (PCF) and a contention-based distributed coordination function (DCF). PCF is a centralized scheme, while DCF is a distributed one. In 802.11 , priority access to the wireless medium is controlled by the application of an inter-frame space (IFS) time between the frame transmissions. To prevent collisions, the transmitter is obliged to wait for the channel to be free for a specified time interval of distributed inter-frame space (DIFS) before sending a frame. If the medium is currently busy or becomes busy during this interval, the transmitter defers the frame transmission until it detects a DIFS At this point, the transmitter selects a random interval referred to as the backoff time to determine the timing for commence transmission. The backoff time is an integer number of slots uniformly chosen from the interval $(0, C W-1)$, where $C W$ is the backoff window, also referred to as the contention window. The backoff number counts down slot-by-slot, and when it reaches zero, the frame is transmitted.

Due to the nature of wireless communications, stations in the network are unable to detect a collision simply by listening to their own transmissions. Therefore, an immediate positive acknowledgment technique is employed to confirm the successful reception of a frame. Specifically, when receiving a frame, the receiver waits for a time interval of short inter-frame space (SIFS) and then transmits an acknowledgment (ACK) to the transmitter, confirming that the frame has been correctly received. The SIFS is deliberately assigned a shorter interval than the DIFS in order to assign the receiving station a higher priority than any other stations waiting to make transmissions. The ACK is only transmitted if the frame is received correctly, and hence if the transmitter does not receive an ACK, it assumes that the data frame must have been lost, and therefore schedules a retransmission. Figure 1 illustrates the basic operations of the distributed coordination function involved in 802.11 protocol [3].

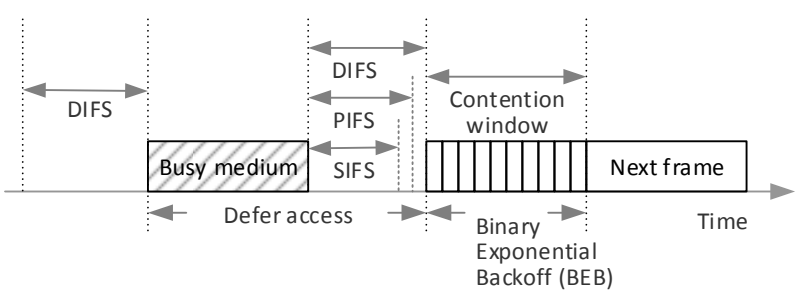

Figure 1. Basic access mechanism in IEEE 802.11 DCF

Legacy IEEE 802.11 MAC has the problem of poor performance because the failure rate of bandwidth competition increases along with the number of mobile station, leading to the degradation of performance. The figure below respectively list the consumed delay time when successfully/failing to transmit packets when basic access mechanism compete channel with virtual carrier-sensing mechanism [4]:


(a) Basic access mechanism

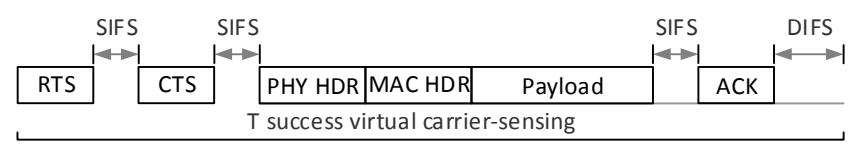

RTS
T collision virtual carrier-sensing

(b) Virtual carrier-sensing mechanism

Figure 2. $T_{s}$ and $T_{c}$ for basic access and virtual carrier-sensing mechanisms 
From Figure 2 it is seen although the implementation of the basic access mechanism is simpler, $T_{c}$ is longer; in comparison, virtual carrier-sensing mechanism has shorter $T_{c}$, but is advantage is its delay time $T_{s}$, transmitting packets would increase greatly. Therefore, although the occurrence of collision decreases, the performance might be influenced due to $\mathrm{RTS} / \mathrm{CST}$ is the extra overhead.

Distributed coordination function (DCF) in IEEE 802.11 [5] utilize CSMA/CA mechanism to conduct media access control. In this scheme, access point and stations continuously monitor the activities of the channel during data transmission/reception; however, this method would cause overhearing, making stations except for transmitter-receiver pair consume a lot of energy to receive unnecessary packet and cause energy waste.

A variety of literatures addressed the selection of Contention Window $(\mathrm{CW})$ has great impact on the efficiency of IEEE 802.11. When CW is large, it would let stations select larger backoff time, and stations would need more time before being able to conduct transmission, leading to delay; when $\mathrm{CW}$ is small, it would let stations select the same backoff time and increase the occurrence of collision. Therefore, large $\mathrm{CW}$ or small CW would both influence the overall efficiency, and thus, a variety of improvement methods for DCF have been derived.

[6] proposed a Bi-Directional Sleep DCF (BDSL-DCF) to improve the energy efficiency of DCF. [7] replaced traditional backoff mechanism by polynomial backoff mechanism, providing improvement method towards real-time transmission to improve the resource competition and access delay. In [8] improved the selection process of contention window (CW) and proposed a method named New Binary Exponential Backoff (N-BEB) which can achieve the fairness and efficiency and reduce packet lost rate. [9] proposed an algorithm which enables every station to dynamically adjust CW size according to channel congestion and accordingly reduce the occurrence of collision.

\section{THE PROPOSED SCHEME}

This study proposed a modified CSMA/CA mechanism of IEEE 802.11 named CSMA/CDA to improve the performance degradation problem confronted in dense WiFi networks. In the proposed scheme, when mobile node transmits data to AP, the mobile node would attach a registered random number (RRN) in the packet. When an AP receives the RRN message piggybacked by data packet, it would add the RRN number to the RRN scheduling list. AP then replies the ACK with an attached RRN information (containing the MAC address of candidate mobile node) to notice the transmitter and the nodes near to the AP. With the proposed scheme, the mobile node which is compatible to CSMA/CA scheme and can utilize the RRN message carried by the AP to update the prescheduling channel information and adjust its backoff timer to reduce the occurrence of collision.

Traditional CSMA/CA mechanism would diagnose the occurrence of collision after the transmission. The proposed scheme can coordinate with AP via RRN message. Partial collision can be avoided before this happens. In the following, the present study will use two examples to explain the difference between CSMA/CA and CSMA/CDA. In the illustrated examples, MH1 and MH2 both have two and three packets to transmit, respectively. The difference between the two examples is mobile node and AP would both piggyback a RRN message to the transmitted packets when the mobile station supports the proposed CSMA/CDA scheme.

In the example of Figure 3, assume the initialized binary exponential backoff time of MH1 andMH2 are 8 and 12, respectively. Owing to the BEB number of $\mathrm{MH} 1$ is shorter, after waiting for (i.e., countdown) 8 slots; MH1 would first acquire the rights for channel access (reselecting a random slot for next channel access from contention window after receiving $\mathrm{ACK}$ ) and then follow the sequence below: $\mathrm{MH} 1, \mathrm{MH} 2, \mathrm{MH} 2$, MH1 and MH2. Refer to Figure 3(b), in this case, collision occurs when MH1 is transmitting second data packet and MH2 is transmitting third data packet at the same slot time, leading to the random backoff procedure is executed due to the occurrence of collision. Table II illustrates the BEB number selected by $\mathrm{MH} 1$ and $\mathrm{MH} 2$.

TABLE II. BEB VARIATION OF MH1 AND MH2 (CSMA/CA)

\begin{tabular}{lll}
\hline Host & Data No. & BEB No. \\
\hline MH1 & 1,2 & $8, \underline{20,10}$ \\
MH2 & $1,2,3$ & $12,10,6,36$ \\
\hline
\end{tabular}

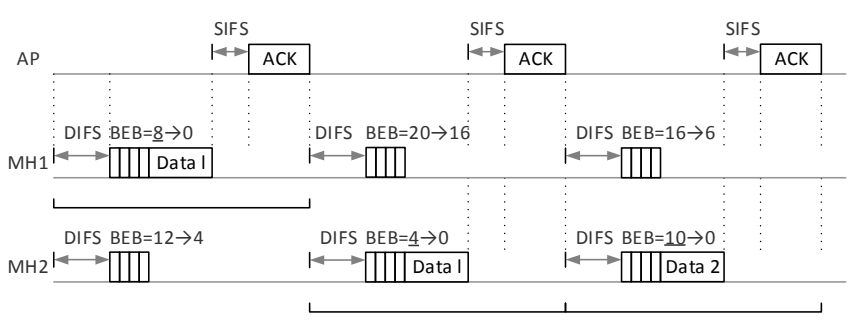

(a) The first half of the transmission period

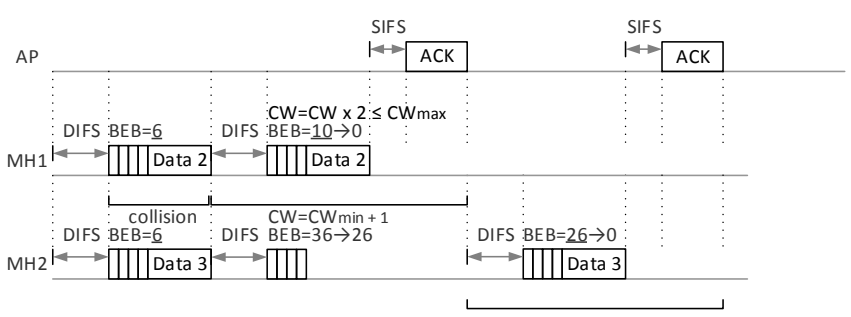

(b) The latter half of the transmission period

Figure 3. Illustration of Legacy CSMA/CA

Similar to Figure 3, Figure 4 also assumes MH1 and MH2 need to transmit 2 packets and 3 packets, where the initialized backoff time is 8 and 12 slots, respectively. In such case, MH1 gets a shorter backoff time and prepares to send the data packet after waiting for 8 slots. With the proposed scheme, MH1 would send the data packet attached by a registered random number $(\mathrm{RRN}=20)$. After MH1 successfully completes packet transmission and passes 4 slot times, MH2 would sequentially send its first and second data packets with the attached RRN, next to the DIFS time. However, it is interesting to notice that 
before MH2 prepares to transmit the third data packet, MH2 notices a collision will occurs after 6 slots and therefore reselects a new random backoff timer. Hence, as shown in Figure $4 \mathrm{~b}$ ), the collision can be avoided compared to the results shown in Figure 3(b).

According to the results shown in Figure 4(c), in comparison with traditional CSMA/CA mechanism (as illustrated in Figure 3), when an AP receives the data packet, it would take out the RRN message and match it with the record in RRN scheduling list to determine whether the slot requested by the transmitter is occupied. After that, AP can select the mobile node which is seen as priority to access the wireless channel sequentially according to RRN scheduling list. After receiving RRN information piggybacked by ACK, the transmitter and the mobile nodes surrounding the AP would avoid to access wireless at the slot time which has been registered by the transmitter to prevent the occurrence of collision. Table III lists the BEB number selected by MH1 and $\mathrm{MH} 2$ with the proposed CSMA/CDA scheme.

TABLE III. BEB VARIATION OF MH1 AND MH2 (CSMA/CDA)

\begin{tabular}{lll}
\hline Host & Data No. & BEB No. \\
\hline $\mathrm{MH} 1$ & 1,2 & $8,20,10$ \\
$\mathrm{MH} 2$ & $1,2,3$ & $12,10,6,36$ \\
\hline
\end{tabular}

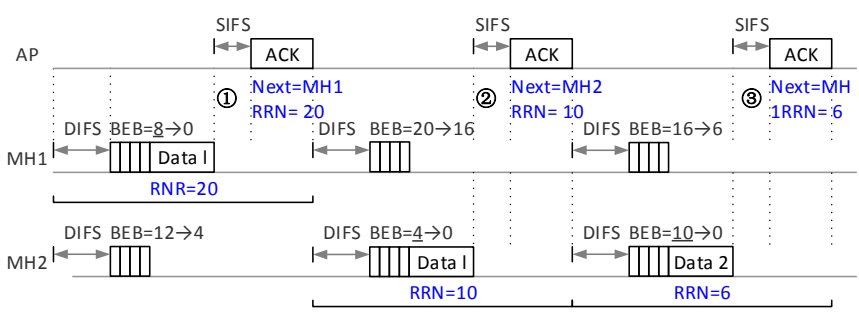

(a) The first half of the transmission period

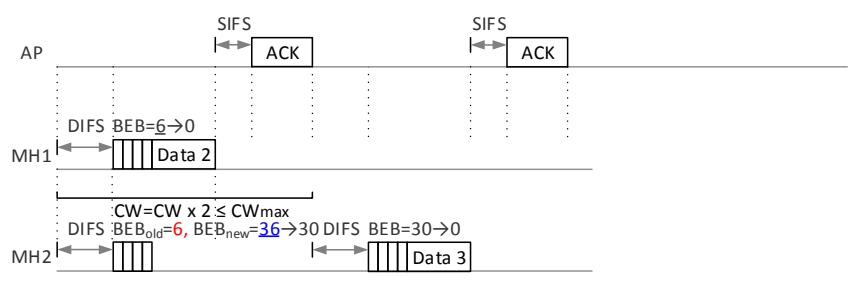

(b) The latter half of the transmission period

\begin{tabular}{|lccc|}
\hline \multirow{2}{*}{ (1) } & Slot & Node & Order \\
\cline { 2 - 4 } & 20 & 1 & 1 \\
\hline \multirow{3}{*}{$(2)$} & Slot & Node & Order \\
\cline { 2 - 4 } & 16 & 1 & 2 \\
& 10 & 2 & 1 \\
\hline \multirow{3}{*}{ (3) } & Slot & Node & Order \\
\cline { 2 - 4 } & 6 & 1 & 1 \\
\hline
\end{tabular}

(c) Example of RRN scheduling lists.
Figure 4. Illustration of the proposed CSMA/CDA

\section{PERFormanCe SimUlation}

This study evaluated the performance of the CSMA/CA and the proposed CSMA/CDA using the developed network simulator based on object-oriented $\mathrm{C}++$. The goals of these tests were to: (1) show the proposed scheme is able to increase the channel access efficiency, and (2) reduce the channel access delay in dense networks by reducing the interference of channel access collision. In the simulations, the mobile nodes are deployed uniformly along the AP. The transmission data rate of the evaluated traffic is $150 \mathrm{Mbps}$. The default values of IEEE 802.11 parameters are listed in Table IV.

TABLE IV. DEFAULT VALUES OF PARAMETERS IN SIMULATIONS

\begin{tabular}{lr}
\hline Physical Meaning & \multicolumn{1}{c}{ Value } \\
\hline SLOT & $9 \mu \mathrm{sec}$ \\
SIFS & $10 \mu \mathrm{sec}$ \\
DIFS & $28 \mu \mathrm{sec}$ \\
PreambleLength & $96 \mathrm{bits}$ \\
PHYhdr & $40 \mathrm{bits}$ \\
$\mathrm{CW}_{\min }$ & 32 \\
$\mathrm{CW}_{\max }$ & 1024 \\
Data frame length & 1000 bytes \\
ACK frame length & 14 bytes \\
\hline
\end{tabular}

Table $\mathrm{V}$ examines the variations of the average network throughput as number of mobile nodes. As shown in Table 5, when there are only 5 to 10 mobile nodes in the network, CSMA/CDA gets higher throughput and complete the data transmission earlier due to the capability to reduce the occurrence of collision; although the average throughput of CSMA/CDA reduces when the number of mobile nodes increases, the throughput of CSMA/CDA throughput has always had better performance. When there are a lot of mobile nodes, e.g. 50 mobile nodes, compared to CSMA/CA, CSMA/CDA can enhance $33.95 \%$ of the throughput. In view of this, it is known CSMA/CDA is beneficial to enhance network performance.

TABLE V. THE THROUGHPUT OF THE VARIOUS MOBILE NODES

\begin{tabular}{ccc}
\hline \# Mobile nodes & CSMA/CDA (Mbps) & CSMA/CA(Mbps) \\
\hline 5 & 7.023928 & 5.985435 \\
10 & 4.121300 & 3.787467 \\
15 & 3.035008 & 2.543127 \\
20 & 2.337861 & 1.806165 \\
25 & 2.042740 & 1.531244 \\
30 & 1.648595 & 1.267646 \\
35 & 1.436737 & 1.008543 \\
40 & 1.274214 & 0.887643 \\
45 & 1.137602 & 0.796546 \\
50 & 1.047915 & 0.677954 \\
\hline
\end{tabular}

As regard to the latency using legacy CSMA/CA and the proposed CSMA/CDA, the results in Table VI show that, compared to CSMA/CA, CSMA/CDA has less delay time because CSMA/CDA can greatly reduce the occurrence of collision, avoiding using larger contention window when there is random backoff; especially when the number of mobile node 
increases. Taking 20 nodes as example, CSMA/CDA reduces $23.12 \%$ of the delay time. When the number of mobile node increases up to 50, CSMA/CDA can reduce $25.04 \%$ of the delay. The simulation results show CSMA/CDA has excellent performance when the number of mobile nodes increases.

TABLE VI. THE DELAY OF THE VARIOUS MOBILE NODES.

\begin{tabular}{ccc}
\hline \# Mobile nodes & CSMA/CDA $(\mathrm{Sec})$ & CSMA/CA $(\mathrm{Sec})$ \\
\hline 5 & 0.002471 & 0.002935 \\
10 & 0.004231 & 0.005210 \\
15 & 0.006043 & 0.007642 \\
20 & 0.007892 & 0.011409 \\
25 & 0.009696 & 0.012394 \\
30 & 0.011932 & 0.015476 \\
35 & 0.013695 & 0.016781 \\
40 & 0.016064 & 0.021056 \\
45 & 0.017913 & 0.024026 \\
50 & 0.019965 & 0.026923 \\
\hline
\end{tabular}

From the above simulation results it is known, CSMA/CDA can effectively reduce the occurrence of collision, enhance throughput and alleviate the delay. Most importantly, it can avoid unnecessary power consumption caused by transmission collision. The results showed the proposed CSMA/CDA scheme outperforms legacy CSMA/CA, especially in dense WiFi networks.

\section{CONCLUSION}

Recent WiFi products enable dramatic improvements in data rates but are still of poor performance in the case of massive mobile stations. In order to solve this problem, the present study proposed a novel media access scheme for nextgeneration WiFi networks. The proposed CSMA/CDA scheme exploits the channel access information collected by associate mobile stations for AP to continuously coordinate the media access across the network. The present study evaluated the performance of the proposed scheme within different operating scenarios. Simulation results reveal that the proposed CSMA/CDA media access control scheme provides higher performance and shorter delay than the CSMA/CA scheme in dense environment of 802.11ax networks.

\section{REFERENCES}

[1] Y. Wang, R. Naylor. Challenges in Designing $5 \mathrm{GHz} 802.11 \mathrm{ac}$ WiFi Power Amplifiers, 2014 IEEE Topical Conference on Power Amplifiers for Wireless and Radio Applications (PAWR), pp. 16 - 18, 19-23 Janne 2014.

[2] D. J. Deng, K. C. Chen, R. S. Cheng, IEEE 802.11ax: Next Generation Wireless Local Area Networks, IEEE 10th International Conference on Heterogeneous Networking for Quality, Reliability, Security and Robustness (Qshine 2014), Rhodes, Greece, August 18-20, 2014.

[3] D. J. Deng and H. C. Yen, Quality-of-Service Provisioning System for Multimedia Transmission in IEEE 802.11 Wireless LANs, IEEE Journal on Selected Areas in Communications, Vol. 23, No. 6, June 2005.

[4] G. Bianchi, Performance Analysis of the IEEE 802.11 Distributed Coordination Function, IEEE Journal on Selected Areas in Communications, Vol. 18, No. 3, March 2000.

[5] IEEE, Part 11: Wireless LAN Medium Access Control (MAC) and Physical Layer (PHY) Specifications, IEEE 802.11 Std., 2012.

[6] R. Palacios, F. Granelli, D. Kliazovich, L. Alonso, J. Alonso-Zarate, An Energy Efficient Distributed Coordination Function Using Bidirectional Transmissions and Sleep Periods for IEEE 802.11 WLANs, 2013 IEEE Global Communications Conference (GLOBECOM), pp. 1619 - 1625, 2013.

[7] S. Bi, Y. J. Zhang, Mitigating Power Law Delays: The use of Polynomial Backoff in IEEE 802.11 DCF, IEEE International Conference on Communications (ICC), pp. 5210 - 2515, 2012.

[8] M. Shurman, B. Al-Shua'b, M. Alsaedeen, M.F. Al-Mistarihi, K. A. Darabkh, N-BEB: New Backoff Algorithm for IEEE 802.11 MAC protocol, 2014 37th International Convention on Information and Communication Technology, Electronics and Microelectronics (MIPRO), pp. $540-544,2014$

[9] K. Hong, S. Lee, K. Kim, Y. Kim, Channel Condition Based Contention Window Adaptation in IEEE 802.11 WLANs, IEEE Transactions on Communications, pp. $469-478,2012$. 